\title{
Optical properties and applications to production of plasmonic thin film nanostructures of self-ordered columnar alumina arrays on glass.
}

\author{
Angus Gentle, Abbas Maaroof, Geoff Smith and Michael Cortie \\ Institute of Nanoscale Technology and Department of Applied Physics, \\ University of Technology, Sydney \\ PO Box 123, Broadway, NSW 2007 Australia
}

\begin{abstract}
In this paper we report on new techniques for making self-ordered porous layers of alumina of varying aspect ratios on glass, without the use of lithographic or masking techniques. Use of RF etching in one of the hole forming steps and also when filling the holes with sputtered metal is shown to be advantageous over additional anodisation. These hole arrays have intrinsically interesting optical responses which will be reported, but their main use is for nano-patterning of subsequent deposited layers either as templates or as masks. High resolution images demonstrate the uniformity in nanohole diameter and in the spacing between holes, which can be achieved when care is used in production.

While many nanostructured materials can be deposited using these Porous Anodic Alumina (PAA) templates we focus here on filling the vertical cylindrical holes with silver. Etching during hole filling leads to better-controlled structures and more efficient processes. Novel optical data on the resultant conducting columnar rings will be presented. Spectrally much sharper plasmon resonant features are found than those reported for classical and more random silver column and island arrays. The optical properties are analysed from an effective medium perspective using data from spectrophotometry and ellipsometry. Fitting this data gives modelled layer thickness and the vertical profile in close agreement with direct SEM imaging. The effective refractive indices of the silver columnar layer have interesting and potentially useful dispersion characteristics.
\end{abstract}

Keywords: porous alumina, optical properties, silver nano-rings, plasmonics, effective medium

\section{INTRODUCTION}

In recent years there has been renewed interest in creating Porous Anodic Alumina (PAA) membranes on Al foils ${ }^{1,2,3}$, with some work carried out on electrically insulating surfaces ${ }^{4}$ such as glass, though the results on glass reported to date have had a relatively disordered PAA structures. We have developed a process for producing ordered PAAs on glass without prior deposition of an underlying conductive layer. PAAs have many potential uses, one of which is for the development of nanostructured metallic/dielectric composites in the form of nanorods or nanorings ${ }^{2}$. This paper details a new way of producing these short range ordered PAAs, along with a comparison between modelled and experimental optical results of Ag filled PAAs. Order here is not long ranged but local. Local order will be seen to be quite strong in terms of uniformity of hole diameter, hole spacing plus the diameter to height aspect ratio of each hole across the complete layer.

In order to develop a controllable method for producing such order in PAAs the current which is passed through them during the anodising process is monitored. Previously acids such as sulphuric, phosphoric and oxalic acids have been used with a range of anodising voltages. Traditionally to create ordered PAAs either imprinting or a two step process of anodisation $^{5}$ was performed in which after an initial anodisation of aluminium is carried out, the surface is then etched away so as to leave a patterned mask, allowing for a second anodisation process to be carried out using the mask from the initial layer as it's starting points. We carry out these steps differently. As we are dealing with thin films of aluminium on glass, a two-step anodisation process was not feasible since a significant quantity of aluminium is usually etched away after the first anodisation. Instead we show that if the initial aluminium film on the glass is of sufficient quality some of the issues arising in use of foils are avoided, such as the cleaning, and etching stages of the process. Details of our processes are described in section 2, including the pore creation steps, and the etching steps.

Photonics: Design, Technology, and Packaging II, edited by Derek Abbott, Yuri S. Kivshar, Halina H. Rubinsztein-Dunlop, Shanhui Fan, Proc. of SPIE Vol. 6038, 603816, (2006) $\cdot 0277-786 \mathrm{X} / 06 / \$ 15 \cdot$ doi: $10.1117 / 12.638126$ 
Section 3 covers the optical data and its modelling with effective medium theory. As we are dealing with a columnar structure, Maxwell Garnet $(\mathrm{MG})^{6}$ and Bruggemann $(\mathrm{BR})^{7}$ effective medium theories with generalised depolarisation factors were tried for both the empty PAA on glass as well as Ag filled PAA on glass for vertical incidence data. Good fitting will be demonstrated for all but the most heavily loaded silver columns with the Bruggemann model. Interesting plasmon resonance effects will be demonstrated, with a shift in resonance wavelength as silver loading increases

\section{EXPERIMENTAL METHOD}

\subsection{Substrate preparation:}

The glass substrate is cleaned by placing it in an ultrasound bath containing Defcon90 cleaning solution for 15 mins. Next it is rinsed with ethanol followed by deionised water and dried with compressed nitrogen and inspected to ensure the substrate is clean, and free from imperfections.

\subsection{Aluminium deposition:}

In order to synthesise ordered porous alumina on glass it is essential that a high quality aluminium film is used in the anodisation process. When the initial aluminium film has a thin oxide layer on top (visually seen as a hazy white on top of the $\mathrm{Al}$ film) the PAA produced becomes less ordered with many unwanted structures included.

In order to achieve high quality $500 \mathrm{~nm}$ thick $\mathrm{Al}$ films e-beam deposition was used. The base pressure of the system was first dropped below $4 * 10^{-6}$ Torr. Out-gassing of the $\mathrm{Al}$ target and chamber was carried out by heating the chamber with a $100 \mathrm{~W}$ halogen lamp for 1 hour. A pre-deposition process was used as follows. A low power setting and current of 250 $\mathrm{mA}$ was used for the e-beam crucible while a shutter shielded the samples. After an initial increase in pressure it would begin to fall back down. Once pressure was below $1.6^{*} 10^{-6}$ Torr the e-beam power was increased to $300 \mathrm{~mA}$ and again we waited for the pressure to drop to below $1.6^{*} 10^{-6}$ Torr. At this point the shutter was opened and the $\mathrm{Al}$ film deposited. The thickness of the Al film was measured using a crystal monitor and confirmed with an Alpha-Step 100 profilometer. In order to stop the film from oxidising due to the heat produced by the e-beam gun, and also to achieve a reduced cool down time before venting, the samples were placed on a water cooled stage which was held at $15^{\circ} \mathrm{C}$.

\subsection{Anodisation:}

Anodisation of the film was carried out with a $40 \mathrm{~V}$ potential in $0.3 \mathrm{M}$ oxalic acid, at a temperature of $2^{\circ} \mathrm{C}$ using a stainless steel counter electrode. A magnetic stirrer was utilised to ensure a uniform temperature in the solution. In order to follow the progress of the anodisation the current flowing through the system was monitored. Locally ordered single step anodisation of the films was possible due to the high quality of the aluminium films deposited.

To ensure complete anodisation of the aluminium conductive copper tape is placed across the top and bottom of the sample with a conducting strip connecting the two on the rear of the sample. $3 \mathrm{M} \# 500$ clear sticky tape is used to cover over these conductive tape areas as well as at the acid/air interface.

The process of anodisation of alumina is summarised in figure 1, in which the different processes are shown. Such current-time characteristic curves agree with those previously reported ${ }^{3,4}$ for PAA growth. 


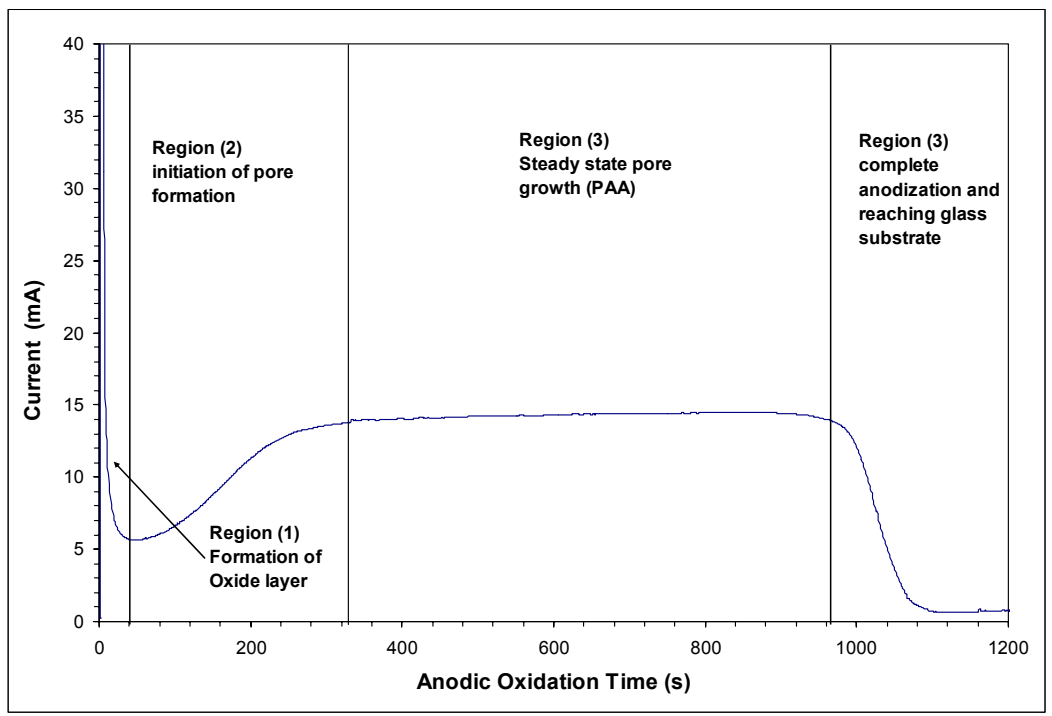

Figure 1: Anodisation Current vs time of a 500nm thick Al film.

\subsection{RF plasma etching}

At this stage the porous anodised layer has quite narrow holes with alumina spacing much larger than hole diameter as in figure 2. The final holes will be much wider in diameter. Before broadening these pores if a particular final aspect ratio is desired it is preferable to etch the upper region of the film away at this point to tune thickness, rather than after broadening. Utilising RF Plasma etching at a rate of $100 \mathrm{~nm} / \mathrm{hr}(3 \mathrm{kV} \mathrm{100W})$ it is possible to first remove these upper layers of the PAA. RF etching has advantages over a chemical step. It allows for the use of aluminium films which are coated in the undesired oxide layer. RF etching through the outer layers has a similar effect as doing a double anodisation (Anodise /Etch /Anodise) previously reported ${ }^{5}$, as it allows for removal of the more disordered outer layer. As RF etching bombards the entire surface of the film pore broadening does not occur while if an acid is used to etch through the outer layer pore broadening can occur. Thus better control is possible. This thickness control intermediate step is optional and included to demonstrate a method for tuning the aspect ratio of columns.

The as anodised alumina on glass film is shown in figure 2. The spacing of the pores is quiet uniform across the substrate though the size of the pores is quite small so the next step is to broaden the pore diameters.

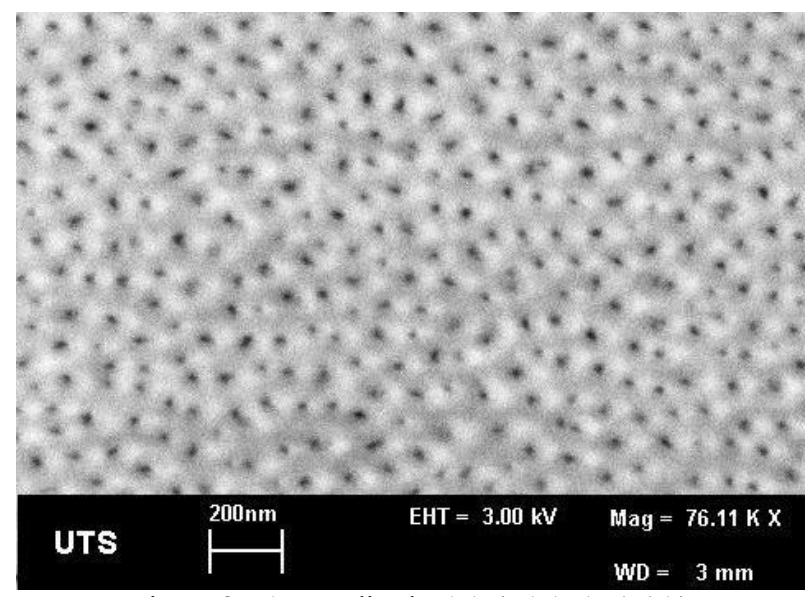

Figure 2 - As anodised PAA (PAA-A71-1A) 


\subsection{Pore broadening}

Pore broadening of the anodised film is carried out in $0.4 \mathrm{M}$ phosphoric acid at room temperature. The optimum time for this step was determined iteratively by first finding the time it takes to entirely dissolve the PAA and working back from there to give an etching time of 1 hour and 15 minutes. The final PAA structure ready for filling with metal is shown in figure 3 and figure 4 . The final cylindrical hole diameters are narrowly dispersed, most being in the range $55 \pm 4 \mathrm{~nm}$.

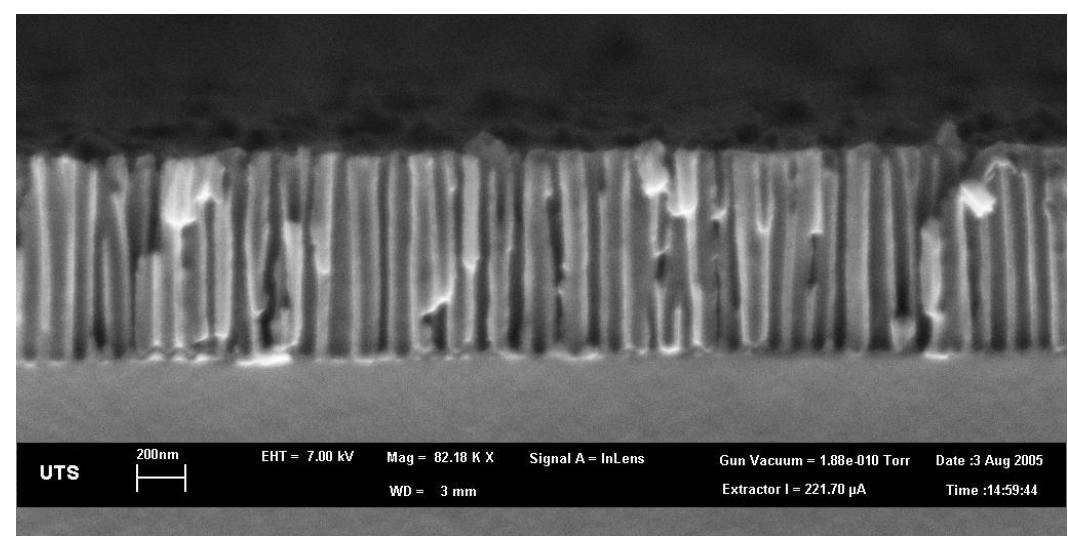

Figure 3: Cross section of PAA structure

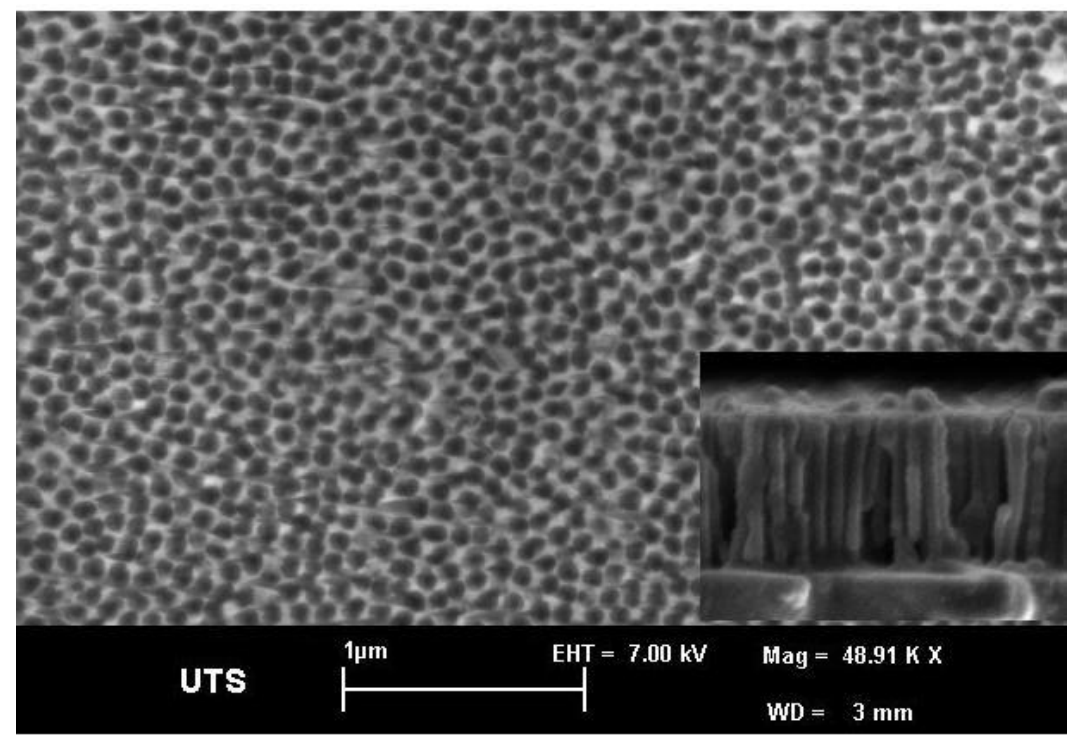

Figure 4: Pore broadened PAA

\subsection{Filling the pores with silver}

Traditional methods for filling anodic alumina have been though an electrolytic process ${ }^{8}$ in which pores are filled with a conducting material initiating at the bottom of the pores, rather than the thin film methods utilized here, which have their filled section in the upper regions of the PAA. The resultant metal structures are also quite different to those produced electrolytically. Filling such small but deep holes under $100 \mathrm{~nm}$ wide and over $600 \mathrm{~nm}$ deep, is not simply a matter of coating. Post metal deposition processing must also be carefully carried out. The pores of the porous alumina film were first partly filled with silver using DC magnetron sputtering to put the equivalent of $60 \mathrm{~nm}$ of Ag onto the PAA. As can be seen from the SEM images of the film in figure 5 (right) not only do the pores contain some silver, but the outer surface is also covered with silver. This top silver layer adds an additional absorption mechanism to the one of interest but it remains specular. Thus it is desirable to etch away the outer surface silver, without removing any silver from the pores, to better control spectral properties. 


\subsection{Nanoring production}

Production of an array of silver nanorings within the alumina structure ${ }^{2}$ without a surface silver layer is feasible through initial deposition of a metal on to the surface and into the pores if followed by a secondary re-sputtering action. We have found that it is possible to incorporate these two steps into one to yield a more efficient process. This is feasible by sputter coating the PAA with silver at the same time as using an RF bias on the substrate to enable simultaneous plasma etching of the upper surface. Best results are obtained by matching the deposition and etch rates such that the etching rate for outer layers of silver is slightly quicker than the deposition rate. This ensures that the silver only remains on the inner walls of the PAA cylindrical voids, and not on the upper surface. It also does not change the overall starting profile, especially the initial total thickness. The silver film is thus deposited under low power with RF bias of $3 \mathrm{kV} 60 \mathrm{~W}$ placed on the sample such that the upper surface is bombarded with argon atoms during deposition. In effect this means $\mathrm{Ag}$ enters the pores but does not remain on the upper surface. The comparison between RF-biased and unbiased Ag coated PAA surface appears in figure 5. To ensure that we were etching all of the surface silver during the RF-biased deposition a glass slide was placed in the chamber. It was next to the PAA and examined with the result being no net deposition of silver on the plain glass slide. Note in figure 5 (left) that the silver partially filled pores remain open. That is the silver has formed nanoparticles in the form of narrow rings lining the pore walls. For the silver volume fractions added (see next section) these rings have quite thin walls ranging from 1 to $4 \mathrm{~nm}$ thick. Our modelling below indicates these very thin rings still behave like continuous reasonably smooth silver, though they are better in this respect after heating.
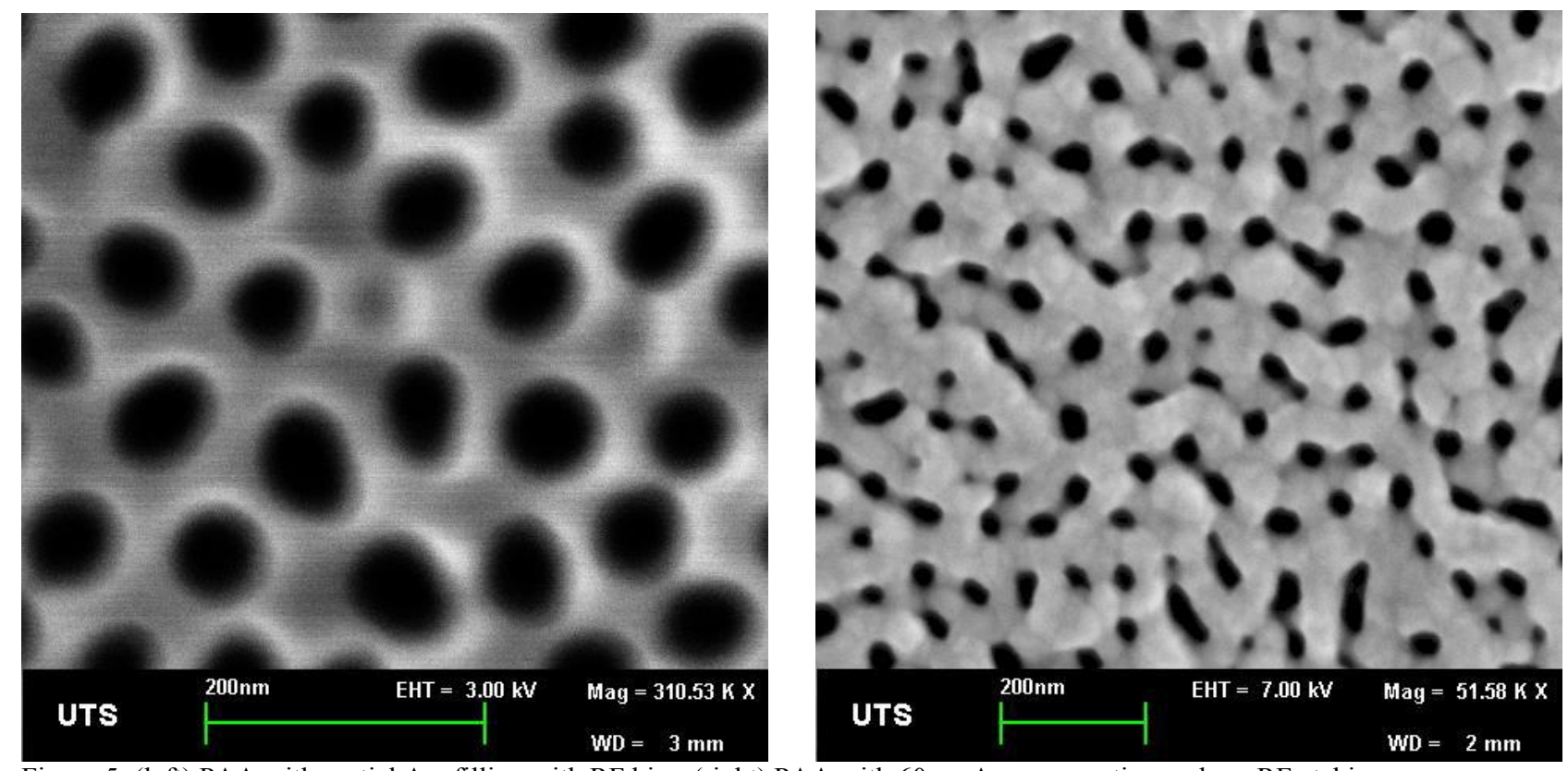

Figure 5: (left) PAA with partial Ag filling with RF bias, (right) PAA with 60nm Ag over coating and no RF etching

The temperature of the sample during the filling process also affects the final silver ring structure and therefore its plasmon resonance. When the sample is prepared at room temperature a broad plasmon absorption resonance is observed. With an increase in temperature a narrowing of the absorption is observed with a tendency to shift from dark brown / purple towards an orange / yellow colour. Alternatively post production annealing of the sample at $400^{\circ} \mathrm{C}$ also yields a narrowing of the resonance, as is seen by a distinct shift from a black/purple colour to a bright yellow colour.

\subsection{The samples to be studied}

A set of four PAA's on glass were produced using the aforementioned method starting from $500 \mathrm{~nm}$ thick high quality aluminium films on glass. Each sample was filled with silver for a different amount of time (1, 2, 3 and 4 minutes). The filling process involved DC magnetron sputtering of silver for a deposition rate of $3.7 \AA / s(I=0.05 \mathrm{~A})$. Simultaneously a $3 \mathrm{kV} 60 \mathrm{~W}$ RF bias is applied to the substrate so as to enable etching of the silver on the upper surface. Next to each sample a glass slide was placed and optically monitored so as to ensure that the etching was carried out at a sufficient rate to stop a build-up of deposited silver developing on the PAA top surface, ensuring silver remains only on the inside 
of the pore walls. A portion of each sample was masked from the filling process so as to allow for measurements to be made on the supporting structure as well as the filled structure.

\section{CHARACTERISATION AND THEORETICAL MODELLING}

\subsection{Optical measurements}

Normal incidence reflectance and transmittance for both bare and filled PAA's were measured utilising a Perkin Elmer Lambda 950 spectrophotometer with Universal Reflectance Accessary, from $300 \mathrm{~nm}$ to $2500 \mathrm{~nm}$. From the spectral $R(\lambda)$ and $\mathrm{T}(\lambda)$ measurements the spectral absorptance $\mathrm{A}(\lambda)$ of the partially filled films was found, as shown in figure 7 .

As can be seen in figure 6 the optical properties of each of the PAA samples before filling with silver are essentially the same which is a good indicator of the degree of quality control in our process. The exception is sample PAA-2 in which a slight extra absorption is seen. This is due to a small amount of residual aluminium left at the alumina/glass interface, which is readily accounted for in the optical modelling.

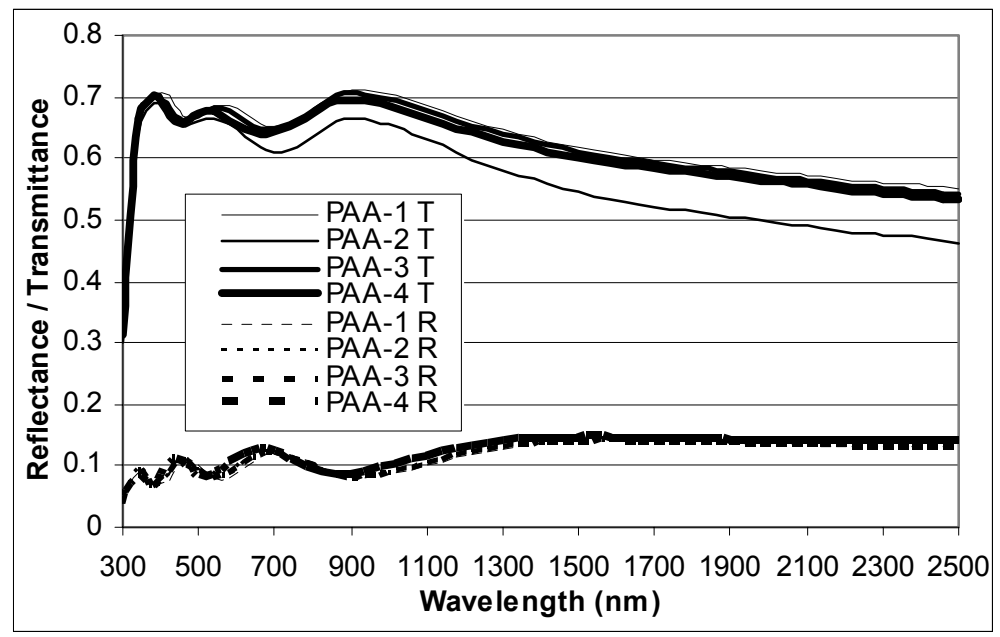

Figure 6: Reflectance and Transmittance for the as anodised PAA's.

The transmittance and absorptance for the four samples in which the identical pore column system has pores part filled with silver under RF bias for varied deposition times are shown in figure 7. Upon close inspection two absorption peaks can be observed in all but the sample with the lowest quantity of silver. The position of the first absorption peak is the most significant and varies between the four samples, being at $435 \mathrm{~nm}, 445 \mathrm{~nm}, 463 \mathrm{~nm}, 490 \mathrm{~nm}$ in order of increasing silver deposition time. This is the plasmon resonant position for the silver rings and will be linked with changes in the effective depolarisation factor of the silver in the columns as the silver filling increases. In the modelling that follows it will be seen that adding more silver does not seem to deepen the filled zone but rather builds up the silver in a given location until the silver volume fraction approaches $14 \%$. That is the ring wall thickness first increases. The second absorption peak is fixed at approximately $500 \mathrm{~nm}$ and is overall film structure related and associated with interference. 

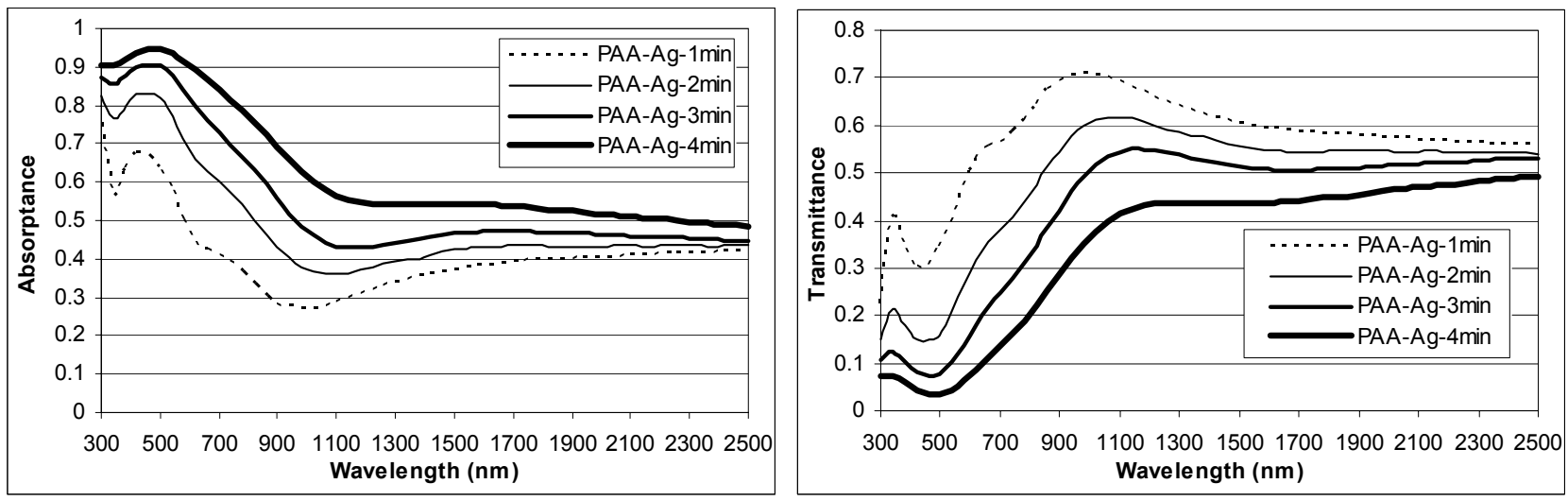

Figure 7: Spectral Absorptance and Transmittance of a series of PAA's partially filled with Silver Rings.

\subsection{Optical Effective Medium Modelling of bare PAA}

As noted earlier both MG and BR models were tried in fitting the optical properties of both the empty and partially filled columnar structure, with up to three component phases in each layer. The MG model gave generally unsatisfactory fits to our data but reasonable fits were found with a generalised BR model. It should be noted that we used them in the form which includes a single generalised depolarisation factor $\mathrm{L}$, not the more commonly used $\mathrm{L}=1 / 3$ for a sphere, when describing the response of the empty columns and those doped with nanoparticles of silver. Such an approach can empirically model the main resonance in a metal ring, though the apparent depolarisation factor needs to be carefully interpreted. For normal incidence properties the relevant depolarisation factor is that for a field perpendicular to the columns. It is this parameter that varies as silver content increases, not the vertical depth of this silver layer. This is due to the silver accumulating where other silver is present, as hollow cylinders ${ }^{2}$ until a high degree of filling is obtained, at which point a more complex and deeper silver structure starts to develop. Evidence for this is seen in the most heavily doped layer which has $16 \%$ by volume of silver and where the simple fitting procedure that works well on the more lightly doped layers, appears to be breaking down.

Firstly the properties of the porous alumina without added silver were determined using a BR effective medium approximation. The final model consisted of four layers as shown below schematically in figure 8 . Layer 0 is the glass substrate. Before $\mathrm{Ag}$ addition we have Layer 1 as a very thin all aluminium layer, layer 2 is also quite thin and contains some Al metal, plus mainly alumina and some voids and layer 3 is the thick columnar porous alumina which is dominated by voids in terms of volume. The modelling with these thicknesses and the compositions shown in table 1 gives a good fit to measured $R(\lambda)$ and $T(\lambda)$ of the undoped films, as can be seen in figure 9 . The $L$ value is common to both components alumina and voids in this BR approximation. Direct observations of the structure with FEGSEM cross section images confirm these optically determined thicknesses to be quite accurate.

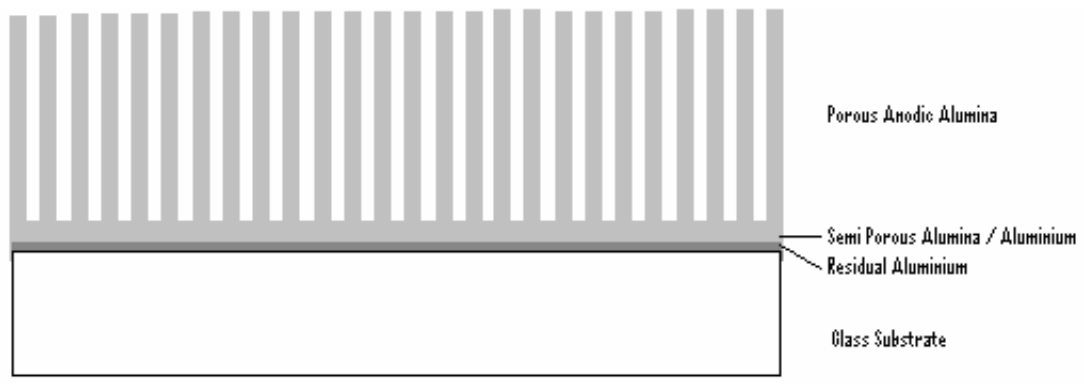

Figure 8: Schematic representation of a three layer bare PAA 


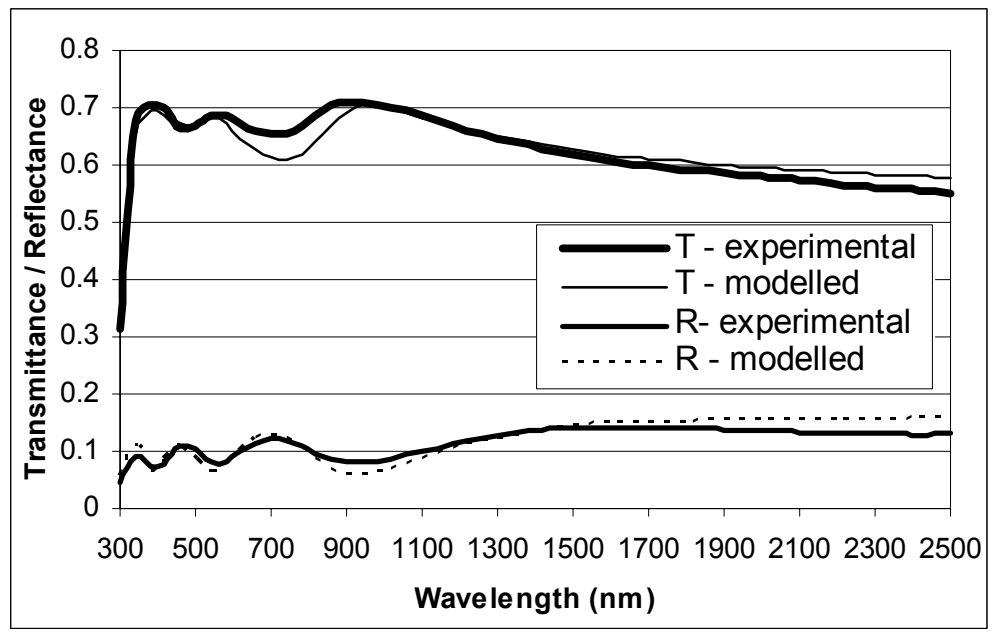

Figure 9: Experimental and modelled optical properties

The porous alumina layer has a high void fraction, thus its effective refractive index is reduced from that of alumina of $\mathrm{n}$ $=1.8-1.7$ to $\mathrm{n}=1.09$. If desired the underlying aluminium in layers 1 and 2 can be removed though heating of the substrate to $400^{\circ} \mathrm{C}$ for 30 minutes so as to oxidise this residual aluminium, thus increasing the transmittance of the entire film. The thickness profile of undoped films in table 1 (layers 0 to 3 ) was found to be exactly repeatable in the four samples made for loading with silver with the only variation being a slight change of up to at most $5 \%$ in the thickness of the upper layer between samples, while all void contents were also identical. We now look at how the effective optical constants of the silver doped layers vary with degree of filling, and how they effect $\mathrm{T}$ and $\mathrm{R}$.

\subsection{Optical Effective Medium Modelling of partially filled PAA}

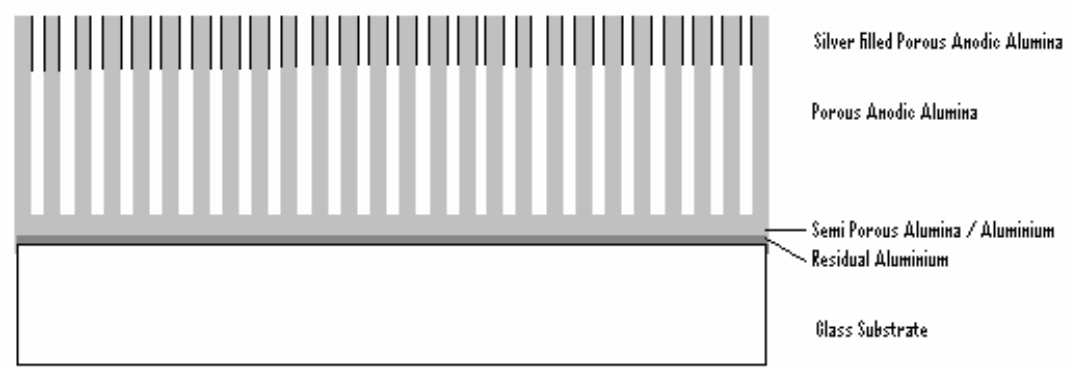

Figure 10: Schematic representation of a five layer partially filled PAA

The doped samples were also modelled with generalised BR theory with 3 phases in the doped layer. The outer layer now has silver, alumina and voids and becomes layer 4 in the stack, while the layer underneath it is as for layer 3 above but the silver free layer 3 is now thinner as part of the original has been partially filled and is now layer 4 . As shown in the schematic in figure 10 the silver rings fill the upper portion of the layer structure only during our dual process, in which all silver is simultaneously removed from the outer layer while it fills the pores. As the deposition time increases initially (table 2) the ring inner diameter first increases followed finally by an increase in ring depth.

Table 1: Partially filled PAA, effective medium and thickness parameters that model optical data

\begin{tabular}{|l|l|l|l|}
\hline Layer \# & $\begin{array}{l}\text { Composition } \\
\text { (Material \%) }\end{array}$ & Thickness (nm) & L factor \\
\hline 4 & Described in Table 2 & & \\
\hline 3 & $\left(\mathrm{Al}_{2} \mathrm{O}_{3} 14 \%\right)($ Void $86 \%)$ & 417 to 444 & 0.2 \\
\hline 2 & $\left(\mathrm{Al} \mathrm{33 \% )}\left(\mathrm{Al}_{2} \mathrm{O}_{3} 56 \%\right)(\right.$ Void $11 \%)$ & 4.66 & 0.33 \\
\hline 1 & Aluminium & 0.641 & \\
\hline 0 & Substrate & $1 \mathrm{~mm}$ & \\
\hline
\end{tabular}


Table 2: Active plasmonic layer thickness and composition (Layer 4)

\begin{tabular}{|l|l|l|l|l|}
\hline $\begin{array}{l}\text { Silver } \\
\text { Deposition } \\
\text { Time (mins) }\end{array}$ & $\begin{array}{l}\text { Composition } \\
(\text { Material \%) }\end{array}$ & $\begin{array}{l}\text { Silver } \\
(\%)\end{array}$ & $\begin{array}{l}\text { Thickness } \\
(\mathbf{n m})\end{array}$ & L factor \\
\hline 1 & $\left(\right.$ Void 83\%) $\left(\mathrm{Al}_{2} \mathrm{O}_{3} 14 \%\right)$ & 3.09 & 140 & 0.21 \\
\hline 2 & $\left(\right.$ Void 78\%) $\left(\mathrm{Al}_{2} \mathrm{O}_{3} 14 \%\right)$ & 7.93 & 150 & 0.27 \\
\hline 3 & $\begin{array}{l}(\text { Void 70\%) } \\
\left(\mathrm{Al}_{2} \mathrm{O}_{3} 14 \%\right)\end{array}$ & 12.9 & 144 & 0.31 \\
\hline 4 & $\begin{array}{l}\left(\mathrm{Void}_{71}\right) \\
\left(\mathrm{Al}_{2} \mathrm{O}_{3} 14 \%\right)\end{array}$ & 15.1 & 180 & 0.30 \\
\hline
\end{tabular}

As can be seen in table 2 for each of the films it is layer 4 (the top layer) which is the active plasmonic absorbing layer due to the varying amounts of silver deposited into the pores of the PAA. The thickness of layer 3 is adjusted to compensate for the reduced thickness of bare PAA, while the underlying films remain unchanged between samples. The silver doped layer in samples 1 to 3, table 2 is approximately constant in thickness.

A comparison between measured and modelled reflectance and transmittance for films with filling time of 2 and 3 minutes are shown in figure 11, as can be seen the modelled data has a reasonable fit to the attained experimental results.
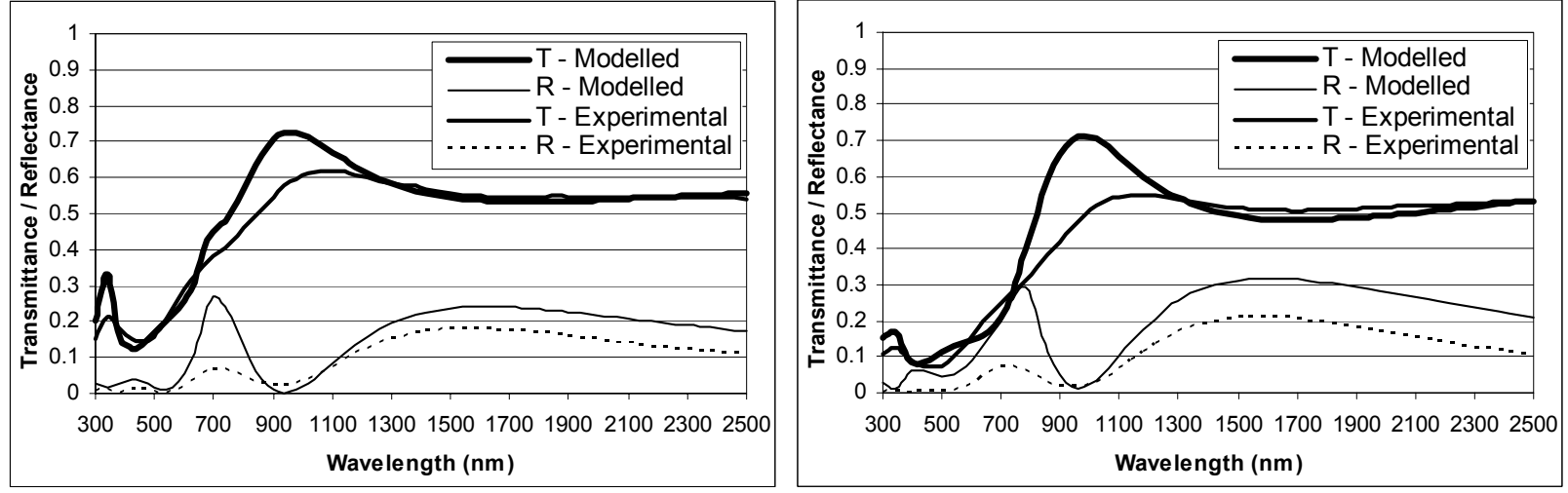

Figure 11: Modelled and Experimental R and T for partially filled PAA. (left) 2min filling, (right) $3 \mathrm{~min}$ filling

\subsection{Effective Optical constants}

The modelled effective refractive index and extinction coefficient for the upper plasmonic layer in this series of filled alumina structures is shown in figure 12. This material has a very interesting optical response. There is a marked difference from the empty porous alumina transmittance and reflectance due to the onset of plasmon resonance. For these columnar ring structures the peak in the resonance as given by the peak in $\mathrm{k}$ and shifts with increasing silver content. This resonant position is governed by the $\mathrm{L}$ factor of table 2 .

A metal ring structure may give a double resonance ${ }^{9}$ according to the two symmetries with which charge can distribute across a thin shell with that with like charges opposite each other on the shell giving the largest total dipole moment for the whole particle and hence the lowest in energy ${ }^{10}$. It is the one of interest here. There is expected to be a shift in resonant energy of a metal ring as ring thickness relative to that of the core changes, just as we observe. The high energy or anti-symmetric resonance does not necessarily exist, or may be very weak, if silver is not sufficiently plasmonic at short wavelengths in this system (due to interband absorption). 


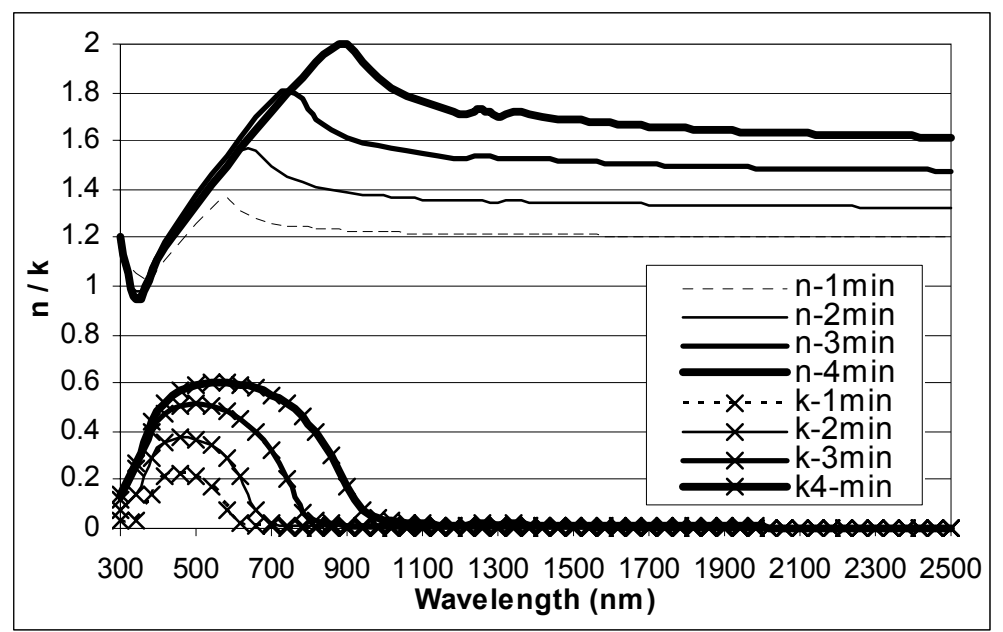

Figure 12: Modelled refractive index of Ag partially filled PAA active layer (Layer 4)

\section{CONCLUSION}

A method for controlled production of plasmonic silver semi-filled porous alumina structures has been reported. The silver forms rings which first grow in wall thickness rather than in depth. The plasmon resonance position shifts as the wall thickness increases. Bruggemann effective medium models of the bare and filled PAA structures have been found to give reasonable fits to optical data provided a three phase layer and a generalised depolarisation factor is used.

\section{ACKNOWLEDGMENTS}

We thank Geoff McCredie of Physics and the staff of the UTS electron microscope unit for assistance.

\section{REFERENCES}

1. G. L. Hornyak, C. J. P., and C. R. Martin, "Fabrication, Characterization, and Optical Properties of Gold Nanoparticle/Porous Alumina Composites: The Nonscattering Maxwell-Garnett Limit", Journal of Physical Chemistry B, 101, 1548-1555, 1997

2. K. L. Hobbs, P. R. Larson, G. D. Lian, J. C. Keay, and M. B. Johnson, "Fabrication of Nanoring Arrays by Sputter Redeposition Using Porous Alumina Templates", Nano Letters, 4, 167-171, 2004

3. G. Q. Ding, M. J.Zheng, W.L. Xu, and W. Z. Shen, "Fabrication of controllable free-standing ultrathin porous alumina membranes", Nanotechnology, 16, 1285-1289, 2005

4. P. G. Miney, P. E. Colavita, M. V. Schiza, R. J. Priore, F. G. Haibach, and M. L. Myrick, "Growth and characterisation of a porous aluminium oxide film formed on an electrically insulating support", Electochemical and solid-state letters, 6 (10) B42-B45, 2003

5. H. Masuda, H. Fukuda, "Ordered metal nanohole arrays made by a two-step replication of honeycomb structures of anodic alumina", Science, 268, 1466-1469, 1995

6. J. C. Maxwell-Garnett, "Colours in metal glasses and in metal films", Philos. Trans. R. Soc. London A, 203, 385420, 1904

7. D. A. G. Bruggemann, "Berechnung verschiedener physikalischer Konstanten von heterogenen Substanzen. I. Dielectrizitätkonstanten und Leitfähigkeiten der Mischkörper aus isotropen Substanzen”, Annalen der Physik, 24, 636-664, 1935

8. C. G. Granquist, A. Anderson, O. Hunderi, "Spectrally selective surfaces of Ni-pigmented anodic $\mathrm{Al}_{2} \mathrm{O}_{3}$ ”, $\mathrm{Applied}$ Physics Letters, 34, 268-270, 1979

9. E. Prodan, C. Radloff, N. J. Halas and P. Nordlander, "A hybridisation model for the plasmon response of complex nanostructures", Science, 302, 419-422, 2003

10. S. Schelm ands G.B. Smith, "Evaluation of the limits of resonance tunability in metallic nanoshells with aspectral averaging technique" J. Opt. Soc. Am. A, 22, 1288-1292, 2005 